\title{
Estimation of Postcardiac Arrest Interval Based on Atrial Cavity Density in Postmortem Computed Tomography
}

\author{
Motoo Yoshimiya, ${ }^{\dagger} \dagger$ Takahiro Ueda,* Tomofumi Ogoshi, ${ }^{*}$ Dawa Zangpo, $\uparrow$ Masato Nakatome, $\uparrow$ and Morio Iino $\dagger$ \\ *Department of Disaster and Emergency Medicine, Tottori University Hospital, Yonago 683-8504, Japan, and †Division of Forensic \\ Medicine, Faculty of Medicine, Tottori University, Yonago 683-8503, Japan
}

\begin{abstract}
Background Intracardiac hypostasis is frequently observed in postmortem computed tomography (PMCT) of acute deaths, and it becomes clearer as the postmortem interval increases. To determine the postcardiac arrest interval (PCAI), we evaluated densities of the right and left atria [anterior part of the right atrium (AR) and posterior part of the left atrium (PL)] using postmortem computed tomographic images.

Methods A total of 184 patients were included in the study. Patients with return of spontaneous circulation and those with final alive confirmation time over 1 hour were excluded. We evaluated the density of AR and PL at the level of the right inferior pulmonary vein entry to the left atrium. We defined the interval between the estimated cardiac arrest time and the postmortem CT time as the PCAI.

Results There was a negative correlation between AR and PCAI in 59 patients who died owing to cardiovascular disease. The regression equation $(\mathrm{PCAI}=-1.725$ $\times \mathrm{AR}+132.95)$ was obtained based on this result. There was no correlation between PL and PCAI.

Conclusion The result suggests that the density of the anterior part of the right atrium decreases as postcardiac arrest interval increases in the case of cardiovascular disease. The regression equation may be used as an additional method to estimate postcardiac arrest interval.
\end{abstract}

Key words chest compression hemodynamics; postcardiac arrest interval; resuscitation

In cases of unwitnessed cardiac arrest, estimating the time of death is one of the required tasks for physicians to complete death certificates. The estimation is based on the judgment of individual doctors using the overall evaluation including body temperature, rigor mortis, and blood test results such as potassium and lactate levels. It is significantly subjective, and there seems to be no objective way to distinguish the cardiac arrest time. We focused on intracardiac hypostasis for an additional method used to determine postcardiac arrest interval (PCAI).

Hypostasis occurs because of gravitational concentration of red blood cells in the vascular system. It can be observed through the skin, with red dark coloration in dependent areas of the body. ${ }^{1}$ It starts to appear from the moment of cardiac arrest, becoming evident after 3 to 4 hours after death. ${ }^{2}$ In postmortem computed tomography(PMCT), fluid-fluid formation can be observed in many cases considering that sedimentation of red blood cells containing hemoglobin increases the density of the posterior part of the vascular system when patients lied in the supine position. In contrary, the anterior part of the vascular system becomes lower in density because of the plasma containing less red blood cells. ${ }^{3-5}$ The contrast becomes stronger until it reaches its plateau. ${ }^{6}$ Fluid-fluid level formation is more evident in cases of acute death. ${ }^{4}$ In acute death, endothelial cells release tissue plasminogen activator. ${ }^{3,5,7}$ In contrast, in cases of prolonged interval death, blood clot is formed within the large vessels and cardiac cavity. ${ }^{8}$ It is an important finding to distinguish between acute and prolonged deaths. As the postmortem interval increases, the intracardiac hypostasis becomes clearer until it reaches plateau. If the effect of chest compression to hemodynamics and hypostasis in the atrial cavity is small, the cardiac arrest interval can be estimated based on the development of hypostasis in PMCT. This study aimed to propose a method of objectively estimating the PCAI based on intracardiac hypostasis in PMCT.

\section{MATERIALS AND METHODS}

This study was approved by the institutional review board of Tottori University Hospital (21A160). We analyzed PMCT findings of 184 patients with cardiac arrest in the out-of-hospital setting who were transported to our emergency room from January 2017 to December 2020. We excluded patients who achieved return of spontaneous circulation (ROSC), patients with severe hypovolemia or blood clot within the aorta or cardiac

Corresponding author: Takahiro Ueda, MD, PhD

taka33chan@gmail.com

Received 2021 November 15

Accepted 2021 December 9

Online published 2022 January 4

Abbreviations: AR, anterior part of the right atrium; PCAI, postcardiac arrest interval; PL, posterior part of the left atrium; PMCT, postmortem computed tomography; ROSC, return of spontaneous circulation; t-PA, tissue plasminogen activator 


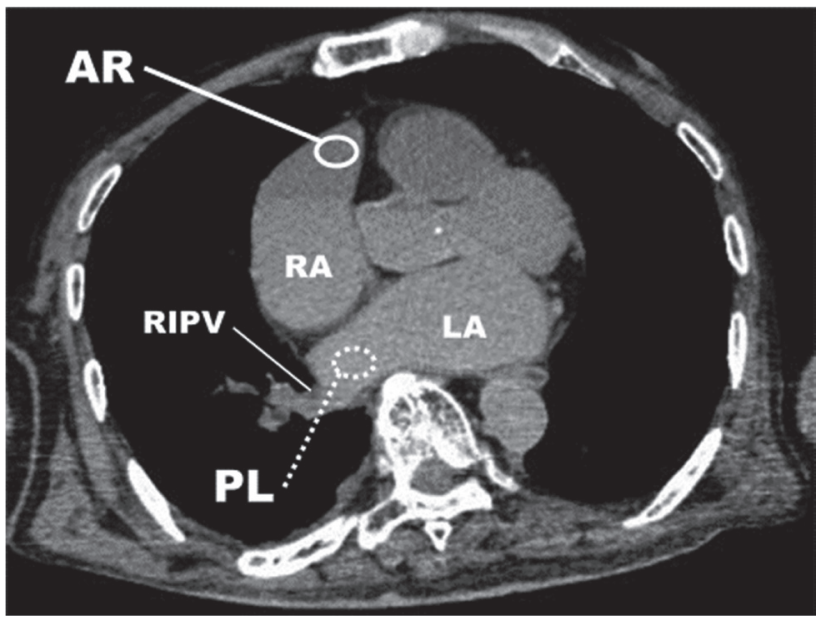

Fig. 1. Measurements are obtained from a circular area with an average size of $100 \mathrm{~mm}^{2}$ in the anterior part of the right atrium (AR) and the posterior part of the left atrium (PL) in an axial view at the level of the right inferior pulmonary vein entrance to the left atrium. AR, anterior part of the right atrium; LA, left atrium; PL, posterior part of the left atrium; RA, right atrium; RIPV, right inferior pulmonary vein.

cavity, and patients with final alive confirmation time over 1 hour of cardiac arrest discovery. All included patients received mechanical (AutoPulse: ZOLL circulation, Sunnyvale, CA, LUCAS ${ }^{\mathrm{TM}}$ : Scanfil Atvidaberg $\mathrm{AB}$, Lund, Sweden) or manual compression or both. The initial cardiac rhythms at the arrival of emergency medical services included asystole, pulseless electrical activity, and ventricular fibrillation.

PMCT was performed using 64-slice Aquilion 64 (Toshiba, Tokyo, Japan) or 256-slice Revolution CT scanner (GE Healthcare, Chicago, IL). We used the axial images of PMCT at the level of the right inferior pulmonary vein entry to the left atrium. The densities of the anterior part of right atrium (AR) and the posterior part of the left atrium (PL) were measured (Fig. 1). For each patient, a circular region of interest (ROI) was defined as an approximately $100 \mathrm{~mm}^{2}$ area in the anterior part of the right atrium and posterior part of the left atrium at the bifurcation level of the right inferior pulmonary vein. We calculated and defined Diff (PL-AR) and Rat (PL/AR) as follows: Diff (PL-AR) $=$ PL-AR, Rat $(\mathrm{PL} /$ $\mathrm{AR})=\mathrm{PL} / \mathrm{AR}$. PCAI was defined as the interval between the estimated cardiac arrest time and the PMCT scanning time. Post-cardiopulmonary resuscitation interval $\left(\mathrm{P}_{\mathrm{CPR}} \mathrm{I}\right)$ was defined as the interval between the resuscitation end time and the PMCT scanning time. For statistical analysis, we used EZR (Saitama Medical Center, Jichi Medical University, Saitama, Japan $)^{9}$ which is R and R commander based freely available statistical software. Pearson's product moment correlation
Table 1. Causes of death

\begin{tabular}{lcc}
\hline Subgroups & $n$ & Ratio (\%) \\
\hline Cardiovascular disease & 60 & 32.6 \\
Asphyxia & 22 & 12.0 \\
Gastrointestinal disease & 5 & 2.7 \\
Cerebrovascular disease & 3 & 1.6 \\
Respiratory disease & 1 & 0.5 \\
Others & 2 & 1.1 \\
\hline Unknown & 91 & 49.5 \\
\hline
\end{tabular}

Cardiovascular disease being the top cause of death, with approximately half being unknown.

coefficient. Normality assumption was verified using the Shapiro-Wilk test.

\section{RESULTS}

A total of 112 males and 72 females were included in the analysis, and their average ages were $85.3 \pm 9.1$ and $75.5 \pm 15.1$ years, respectively. According to the t-test, females were significantly older than males $(t=4.927$, $\mathrm{df}=182, P<0.001)$. The causes of death are listed in Table 1. Cardiovascular disease (e.g., aortic dissection, congestive heart failure, aortic aneurysm rupture) was the leading cause, followed by asphyxia (e.g., bathtub drowning, suicidal hanging, choking). Causes of death were unknown for more than half of the patients. There were 59 patients with cardiovascular disease being the cause of death, including 31 males with an average age of 76.1 years and 28 females with an average age of 85.7 years. The average PCAI was $105 \pm 37$ minutes. Normality assumption was verified for PCAI, AR, and PL. AR $(r=-0.24, P<0.01)$ and PL $(r=0.10, P=0.18)$. The relationship between PCAI and AR, PL, Diff (PLAR), Rat (PL/AR) are shown in Figs. 2-5. PCAI did not correlate with AR, PL. Diff (PL-AR) and Rat (PL/AR) also did not correlate with PCAI $(r=0.23, P<0.01 ; r=$ 0.23, $P<0.01)$.

Considering only 59 patients who died owing to cardiovascular disease, the relationship between PCAI and AR, PL, Diff (PL-AR), Rat (PL/AR) are shown in Figs. 6-9. AR negatively correlated with PCAI ( $r=$ $-0.44, P<0.01$ ). The following regression equation was obtained: PCAI $=-1.725 \times$ AR +132.95 PL, Diff (AR/ $\mathrm{PL})$, and Rat (PL/AR) did not correlate with PCAI $(r=$ $0.01, P=0.93 ; r=0.24, P=0.06 ; r=0.36, P<0.01)$.

The average $\mathrm{P}_{\mathrm{CPR}} \mathrm{I}$ was $27( \pm 17.6)$ minutes. No correlation was found between $\mathrm{P}_{\mathrm{CPR}} \mathrm{I}$ and $\mathrm{AR}(r=-0.21, P$ $=0.10)$ or PL $(r=0.04, P=0.78)$. The hemoglobin level of the blood that was obtained during resuscitation also showed no correlation between $\operatorname{AR}(r=0.07, P=0.60)$ and PL $(0.27, P=0.03)$. 


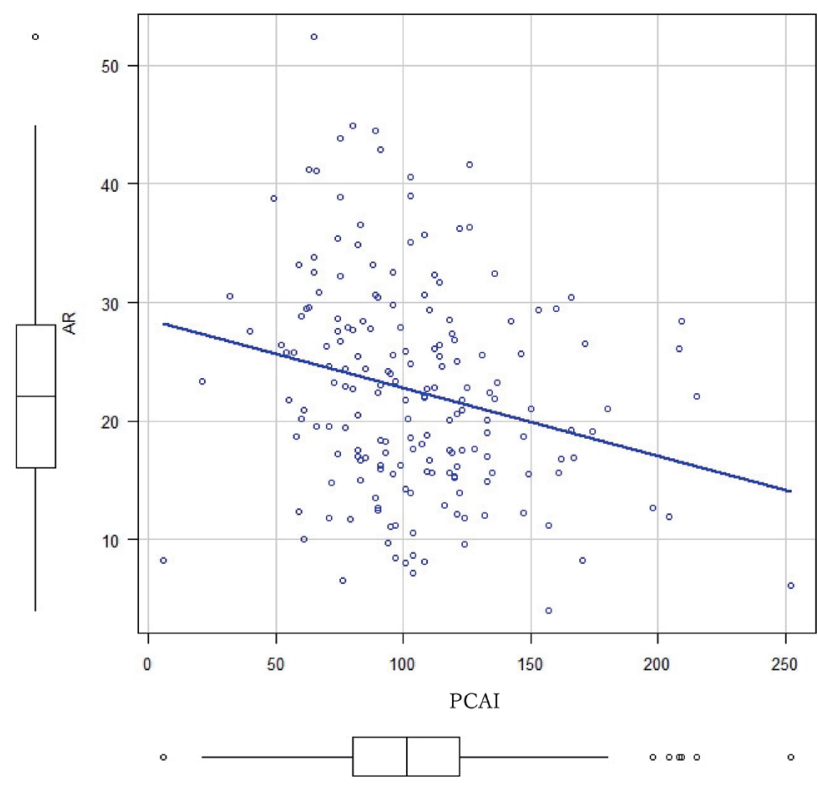

Fig. 2. Correlation between AR and PCAI including all the causes of death. There was no statistical correlation between AR and PCAI. AR, anterior part of the right atrium; PCAI, postcardiac arrest interval.

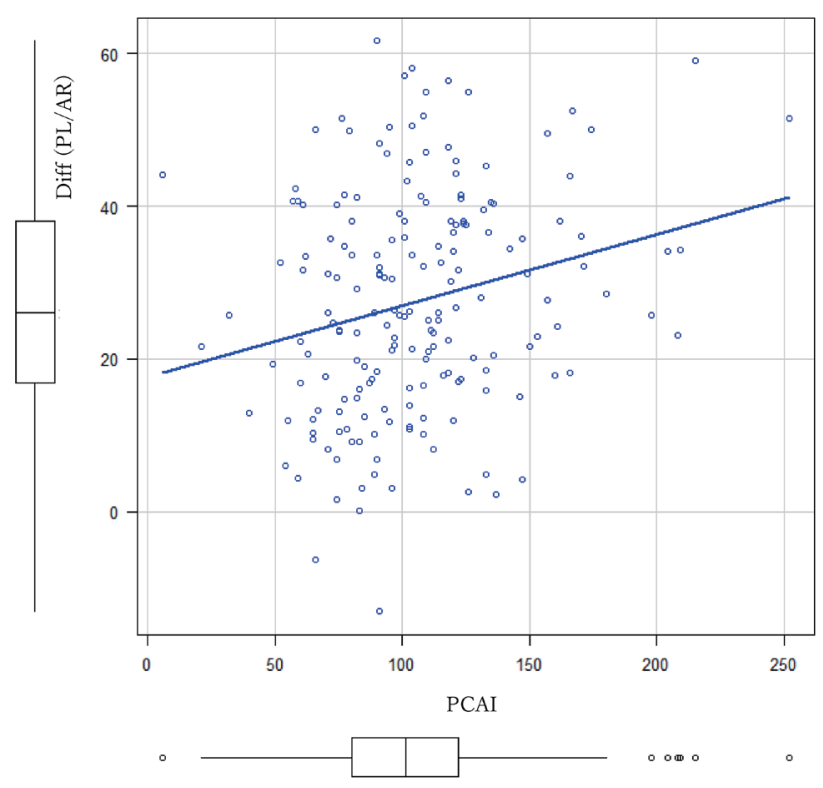

Fig. 4. Correlation between Diff (PL-AR) and PCAI including all the causes of death. There was no statistical correlation between Diff (PL-AR) and PCAI. AR, anterior part of the right atrium; PCAI, postcardiac arrest interval; PL, posterior part of the left atrium.

Considering 22 patients with asphyxia, and 91 patients with unknown cause of deaths, PCAI did not correlate with AR or PL (asphyxia: $r=-0.18, r=0.06$;

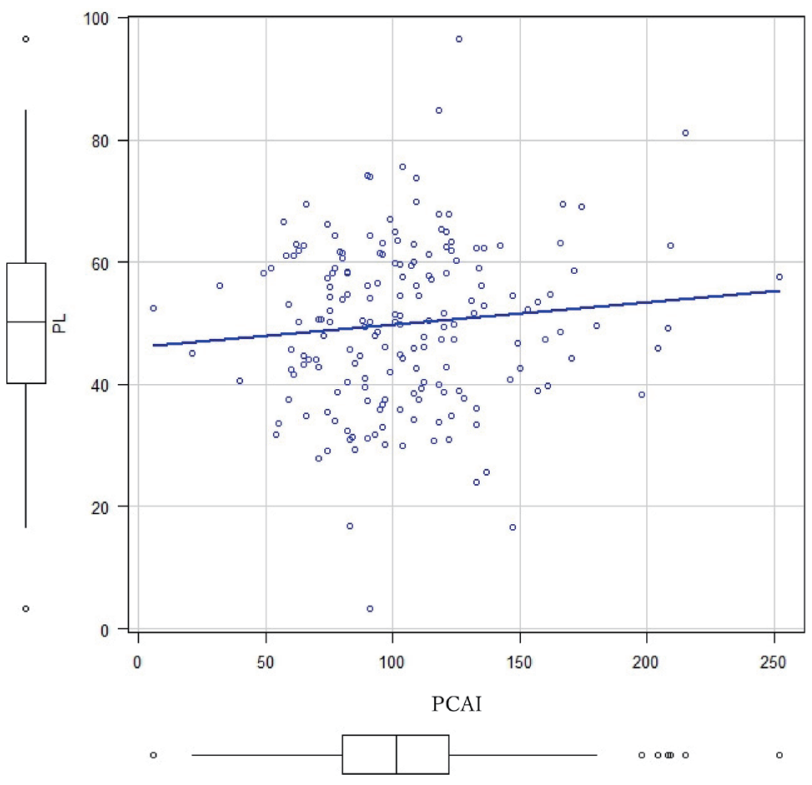

Fig. 3. Correlation between PL and PCAI including all the causes of death. There was no statistical correlation between PL and PCAI. PCAI, postcardiac arrest interval; PL, posterior part of the left atrium.

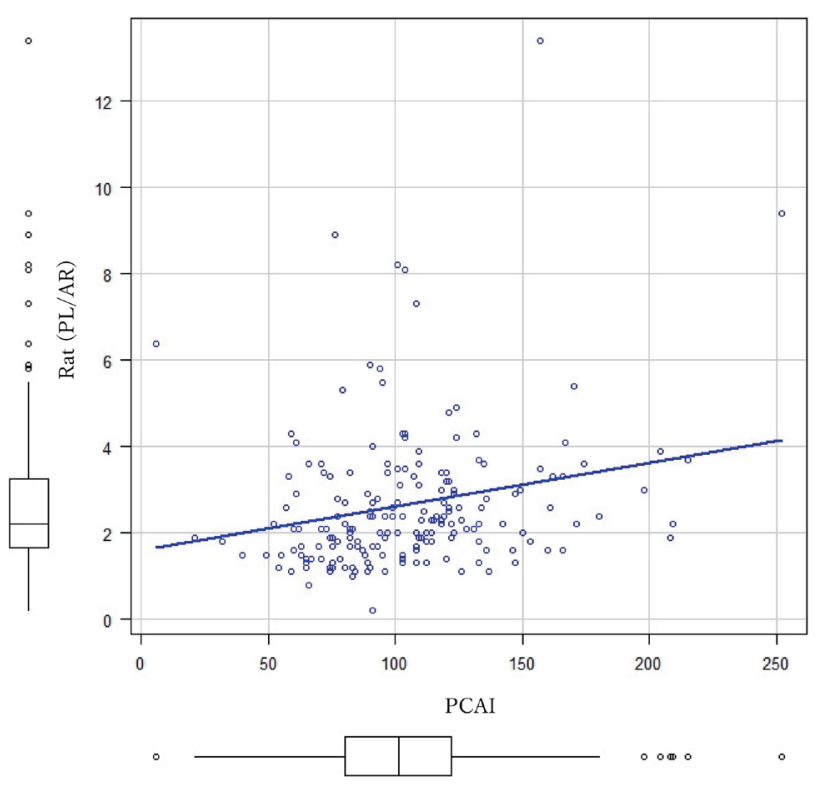

Fig. 5. Correlation between Rat (PL/AR) and PCAI including all the causes of death. There was no statistical correlation between Rat (PL/AR) and PCAI. AR, anterior part of the right atrium; PCAI, postcardiac arrest interval; PL, posterior part of the left atrium.

unknown: $r=0.18, r=0.12$ ). 


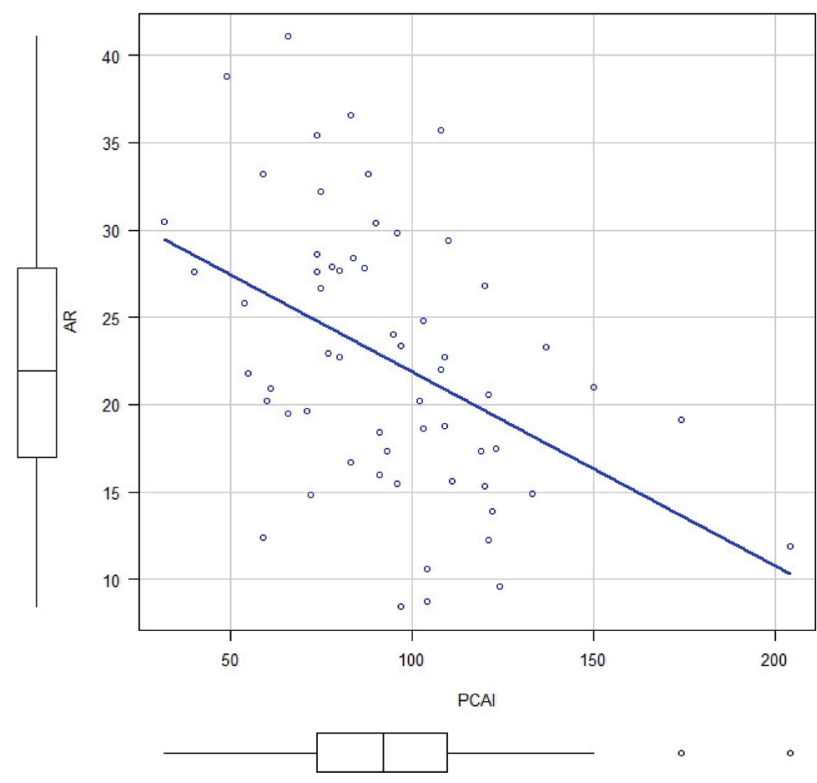

Fig. 6. Correlation between AR and PCAI of patients who died owing to cardiovascular disease. There was a significant negative correlation between AR and PCAI. AR, anterior part of the right atrium; PCAI, postcardiac arrest interval.

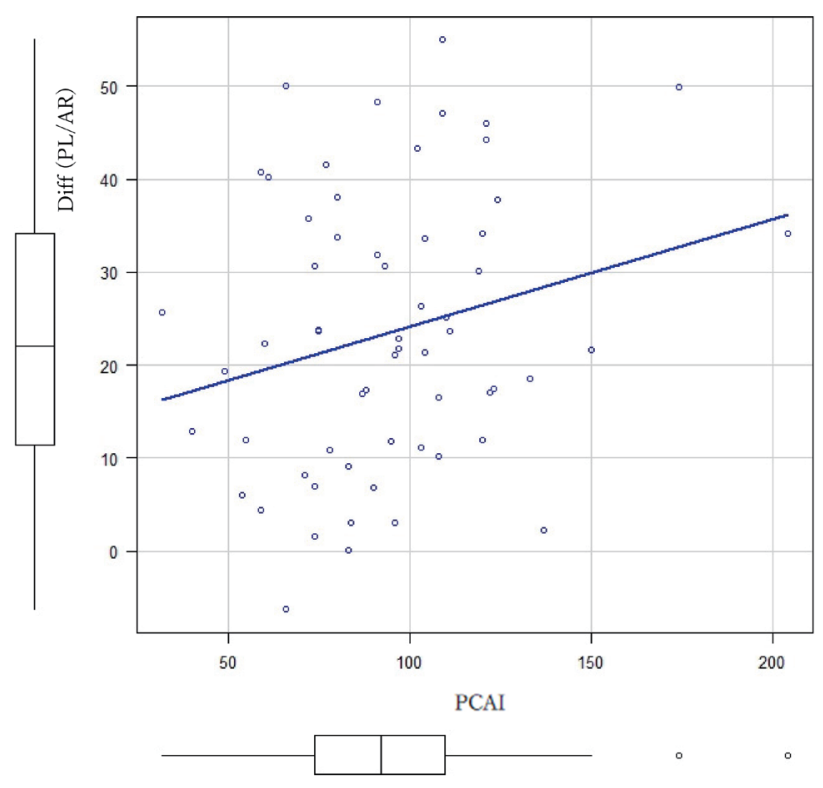

Fig. 8. Correlation between Diff (PL-AR) and PCAI of patients who died owing to cardiovascular disease. There was no correlation between Diff (PL-AR) and PCAI. AR, anterior part of the right atrium; PCAI, postcardiac arrest interval; PL, posterior part of the left atrium.

\section{DISCUSSION}

We found the density of anterior part of the right atrium (RA) decreases as post-cardiac arrest time (PCAI) becomes longer in patients who died of cardiovascular

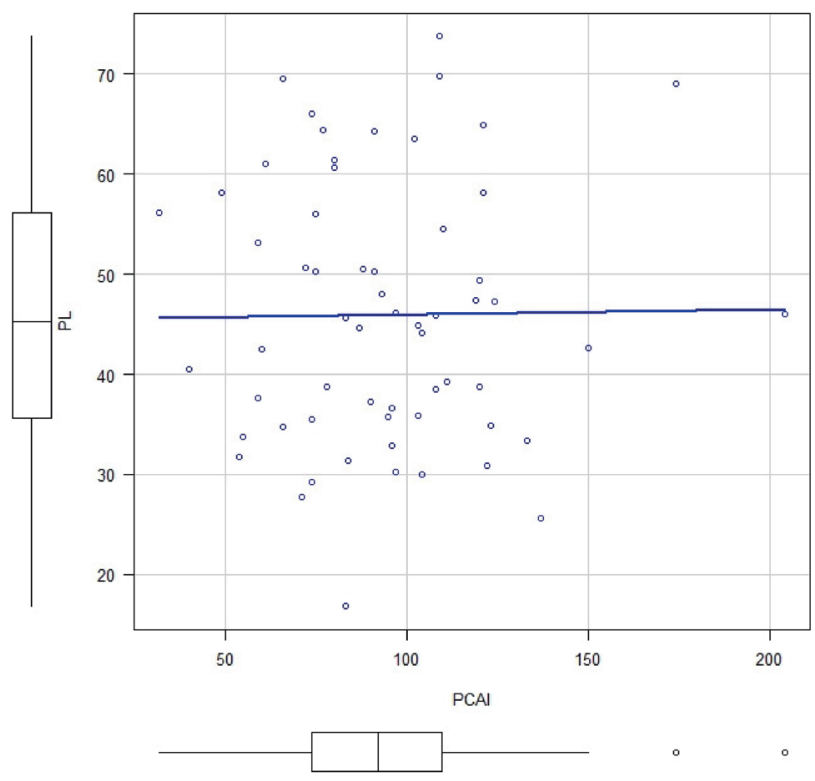

Fig. 7. Correlation between PL and PCAI of patients who died owing to cardiovascular disease. There was no correlation between PL and PCAI. PCAI, postcardiac arrest interval; PL, posterior part of the left atrium.

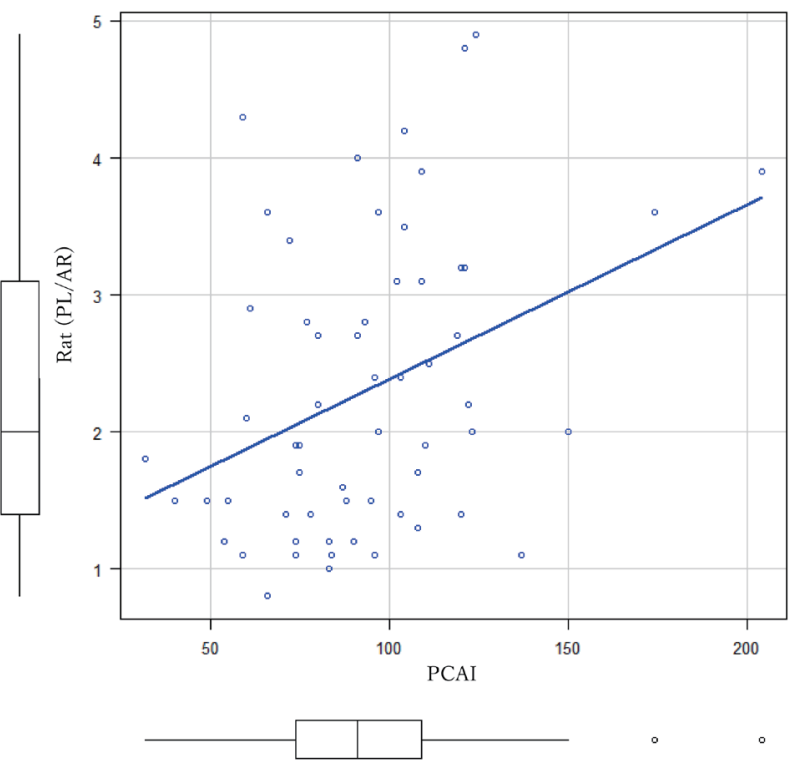

Fig. 9. Correlation between rat (PL/AR) and PCAI of patients who died owing to cardiovascular disease. There was no correlation between rat (PL/AR) and PCAI. AR, anterior part of the right atrium; PCAI, postcardiac arrest interval; PL, posterior part of the left atrium.

disease. Although there seemed to be no fluid-fluid formation in the cardiac cavity, there was an attenuation decrease in RA. Fluid-fluid formation is a subjective finding; thus, it is not possible to estimate the PCAI 
depending on the presence of fluid-fluid level. Instead, the measurement of the density is needed for the interval estimation. We obtained the regression formula $\mathrm{PCAI}=-1.725 \times \mathrm{AR}+132.95$. Using AR density of PMCT performed in emergency field, we could provide assumption regarding the postcardiac interval.

Shiotani has reported that hypostasis is observed in more than half of the bodies that have less than 2 hours of postmortem interval. ${ }^{3}$ In some of our cases, although there is no fluid-fluid level formation, the densities of AR and PL were different. It could be hypothesized that the hypostasis or changes in the density start from the early stages. Ishida et al. have analyzed 31 patients who were transported to the hospital but did not respond to resuscitation and found hypostasis in $55 \%$ of the patients. ${ }^{10}$ They observed hypostasis in the right atrium, left atrium, and thoracic aorta.

Zerbini et al. performed multiple PMCT over time for 23 individuals who died of natural causes under medical care and measured the density of RA and PL. ${ }^{6}$ As a result, the difference between AR and PL increased as the interval became longer. They have concluded that in patients within 12 hours of postmortem interval, the time of death with the range of 2 hours could be estimated. ${ }^{6}$ However, it is not practical to perform PMCT multiple times without moving the body from the CT stage. In many facilities in Japan, they would only perform one native $\mathrm{CT}$ as a postmortem imaging.

During resuscitation with chest compression, cardiac output is decreased to $10-30 \%$ of normal status. ${ }^{11}$ If the effect of chest compression to the hypostasis is limited, cardiac arrest estimation using RA density is reasonable.

Although there was no significant correlation among AR, PL, Diff (PL-AR), and Rat (PL/AR), including all the causes of death, people who died owing to cardiovascular disease showed negative correlation between AR and PCAI. The amount of intrinsic tissue plasminogen activator (t-PA) release could be different according to the cause of death. The cardiovascular disease included impairment of vessels such as aortic dissection or aneurysm rupture. This might affect the amount of t-PA released from the endothelial wall; thus, when there is insufficient intrinsic t-PA, hypostasis may not be observed in the early stage of the postmortem period. Buchtele et al. have reported that increased fibrinolytic system would predict poor prognosis ${ }^{12}$ which seems to be consistent with our results that non ROSC cases in cardiovascular diseases showed correlation between RA and PCAI. The fact that only the cases with cardiovascular cases showed correlation also suggests that blood viscosity differs depending on the cause of death. Contrary to our expectations, there was no correlation between PL and PCAI. The density of PL is expected to be affected by various factors including hematocrits and coagulation states. In a case with a low number of hematocrits in the antemortem state, we assume that PL would not get higher than a case with a high number of hematocrits.

Although all the patients received resuscitation with chest compression, AR negatively correlated with PCAI. This chronological decreased of AR suggests that hypostasis starts from the moment of cardiac arrest. Possibly, blood flow is not circulating as in a manner of spontaneous cardiac movement. Hemodynamics during mechanical chest compression has been reported by Hansen et al. using time-resolved volumetric CT. ${ }^{13}$ According to them, the atria are thought to be contracted well, pushing against the sternum and vertebrae. However, ejection fraction and cardiac output during chest compression remain unknown. In our study, PL did not correlate with PCAI; thus, the left atrium might have more blood flow inside the cavity.

Cheskes et al. have evaluated the association between chest compression fraction (CCF) and survival to hospital discharge, ROSC, and neurologically intact survival. ${ }^{14}$ They concluded that CCF was paradoxically associated with lower odds of survival and may not be a predictor of good clinical outcomes. Gates et al. have reported that the mechanical chest compression device did not show an advantage for survival to discharge/30 days, survival with good neurological outcome, compared to manual chest compression. ${ }^{15}$ These results suggest that blood flow during chest compression is insufficient. Some studies have stated that chest compression is effective for resuscitation ${ }^{16}$; thus, further studies are necessary.

According to Zerbini et al.'s study, the decline of AR density might have the equilibrium. ${ }^{6}$ Our study included patients with PCAI less than 4 hours and 12 minutes; thus, the equation may not be applicable in patients with longer PCAI. In our study, cardiac arrest included asystole, pulseless electrical activity, and ventricular fibrillation. It is unclear how different waveforms affect AR density decline. This study was conducted at a single-center tertiary emergency hospital in Yonago, located in the relatively rural area in Japan with a population of 150 thousand.

As reported in previous studies, some early postmortem changes could be observed on an hourly basis, but some will appear on a daily basis. Ishikawa et al. have reported aorta narrowing just below the diaphragm starts from 12 hours after death and continues to increase within 5 days. ${ }^{17}$ Decreased aerated lung volume 
and rate have been reported as an early postmortem change. ${ }^{18}$ Increased rate of pleural-space-fluid collection increases 30 hours after death. ${ }^{18}$ Our study revealed that decreased density of the RA after cardiac arrest seems to be a significant early change in cardiovascular death, so this phenomenon is thought to be stabilized within a few hours after cardiac arrest. According to Zerbini et al., the hypostatic change seems to stabilize 12 hours after death; thus, the density of the RA will reach its plateau in similar time course. ${ }^{6}$ The PCAI in our study ranged from 21 to 252 minutes (32-204 minutes for cardiovascular disease); estimation using our method is limited to patients with PMCT performed within 4-5 hours after cardiac arrest. Our findings suggest the change starts not from the time of death but from the moment of cardiac arrest. It is important to recognize the time course of death and combine different findings when estimating the time of death. One limitation of our study is there was only one measurer of ROI, so there might be certain measurement errors and biases. Another limitation is that the position at the time of discovery is unknown and might affect the density in the cardiac cavity.

In conclusion, we evaluated the density within the atrial cavity of PMCT and its association with PCAI. AR showed significantly negative correlation with PCAI only in patients with cardiovascular disease. This result indicates that hypostasis in the right atrium starts from the time of cardiac arrest, and the effect of chest compression to the right atrium is limited. This negative correlation is only found in patients with cardiovascular diseases. The regression equation obtained from this result may be used as an additional method to estimate the PCAI in patients with cardiovascular disease.

The authors declare no conflict of interest.

\section{REFERENCES}

1 DiMaio D, DiMaio V. Forensic Pathology. Second. Boca Raton, FL: CRC Press; 2001.

2 Dolinak D, Matshes E, Lew EO. Forensic Pathology; Principles and practice. Burlington, VA: Academic Press; 2005.

3 Shiotani S, Kohno M, Ohashi N, Yamazaki K, Itai Y. Postmortem intravascular high-density fluid level (hypostasis): CT findings. J Comput Assist Tomogr. 2002;26:892-3. DOI: 10.1097/00004728-200211000-00006, PMID: 12488731

4 Takeichi S, Wakasugi C, Shikata I. Fluidity of cadaveric blood after sudden death. Am J Forensic Med Pathol. 1984;5:223-8. DOI: 10.1097/00000433-198409000-00006, PMID: 6496435

5 Takeichi S, Wakasugi C, Shikata I. Fluidity of cadaveric blood after sudden death: part II. Am J Forensic Med Pathol. 1985;6:25-9. DOI: 10.1097/00000433-198503000-00006, PMID: 4039106
6 Zerbini T, da Silva LFF, Lobato Baptista PA, Ikari ES, Rodrigues de Araujo M, de André CDS, et al. Estimation of post mortem interval by tomographic images of intra-cardiac hypostasis. J Forensic Leg Med. 2016;38:111-5. DOI: 10.1016/ j.jflm.2016.01.001, PMID: 26802976

7 Nakasono I. A study on the fibrinolytic activity of cadaveric blood: isolation and characterization of plasminogen activator from cadaveric plasma after sudden death. Nippon Hoigaku Zasshi. 1984;38:280-8. PMID: 6541262

8 O'Donnell C, Woodford N. Post-mortem radiology—a new sub-speciality? Clin Radiol. 2008;63:1189-94. DOI: 10.1016/ j.crad.2008.05.008, PMID: 18929036

9 Kanda Y. Investigation of the freely available easy-to-use software 'EZR' for medical statistics. Bone Marrow Transplant. 2013;48:452-8. DOI: 10.1038/bmt.2012.244, PMID: 23208313

10 Ishida M, Gonoi W, Hagiwara K, Takazawa Y, Akahane M, Fukayama M, et al. Hypostasis in the heart and great vessels of non-traumatic in-hospital death cases on postmortem computed tomography: relationship to antemortem blood tests. Leg Med. 2011;13:280-5. DOI: 10.1016/j.legalmed.2011.09.004, PMID: 22000062

11 Duggal C, Weil MH, Gazmuri RJ, Tang W, Sun S, O’Connell F, et al. Regional blood flow during closed-chest cardiac resuscitation in rats. J Appl Physiol. 1993;74:147-52. DOI: 10.1152/jappl.1993.74.1.147, PMID: 8444685

12 Buchtele N, Schörgenhofer C, Spiel AO, Jilma B, Schwameis M. Increased Fibrinolysis as a Specific Marker of Poor Outcome After Cardiac Arrest. Crit Care Med. 2018;46:e9951001. DOI: 10.1097/CCM.0000000000003352, PMID: 30096099

13 Hansen K, Machin R, James J, Coats T, Rutty GN. A look inside cardiopulmonary resuscitation: A 4D computed tomography model of simulated closed chest compression. A proof of concept. Resuscitation. 2020;153:149-53. DOI: 10.1016/j.resuscitation.2020.05.037, PMID: 32526250

14 Cheskes S, Schmicker RH, Rea T, Powell J, Drennan IR, Kudenchuk P, et al.; Resuscitation Outcomes Consortium investigators. Chest compression fraction: A time dependent variable of survival in shockable out-of-hospital cardiac arrest. Resuscitation. 2015;97:129-35. DOI: 10.1016/ j.resuscitation.2015.07.003, PMID: 26232514

15 Gates S, Quinn T, Deakin CD, Blair L, Couper K, Perkins GD. Mechanical chest compression for out of hospital cardiac arrest: systematic review and meta-analysis. Resuscitation. 2015;94:91-7. DOI: 10.1016/j.resuscitation.2015.07.002, PMID: 26190673

16 SOS-KANTO study group. Cardiopulmonary resuscitation by bystanders with chest compression only (SOS-KANTO): an observational study. Lancet. 2007;369:920-6. DOI: 10.1016/S0140-6736(07)60451-6, PMID: 17368153

17 Ishikawa N, Nishida A, Miyamori D, Kubo T, Ikegaya H. Estimation of postmortem time based on aorta narrowing in CT imaging. J Forensic Leg Med. 2013;20:1075-7. DOI: 10.1016/j.jflm.2013.09.026, PMID: 24237823

18 Hyodoh H, Shimizu J, Watanabe S, Okazaki S, Mizuo K, Inoue H. Time-related course of pleural space fluid collection and pulmonary aeration on postmortem computed tomography (PMCT). Leg Med. 2015;17:221-5. DOI: 10.1016/ j.legalmed.2015.01.002, PMID: 25657038 